\title{
Analysis on the Low Voltage Electrical Switch Contact Performance and Design
}

\author{
Wang Bao-ying ${ }^{1, a}$ \\ ${ }^{1}$ Chongqing College of Electronic Engineering, Chongqing, 401331, China \\ awangbaoying401331@163.com
}

Keywords: Low voltage electrical switch; Contact performance; Design

\begin{abstract}
The role of the electric switch has two aspects: the first is the function of the switch itself. It is the tube and off of electric circuit and short circuit or over current that can be automatically jump to cut off the power supply and it has the effect of protection circuit and electric equipment. The second is the social function that people in today's life can not be lack. It can give people's work, study and life convenient, also can provide insurance coefficient. Thus, once it has quality problems, it will directly affect the quality of people's lives. Generally, the control class of low voltage electrical equipment mainly includes control relay and starter and other electrical equipment, power distribution type of low voltage electrical equipment mainly includes circuit breaker and switch control. Against such a situation, this article will focus on the performance analysis and design of low voltage electrical switch contact.
\end{abstract}

\section{Introduction}

The switching power supply is through advanced power electronics technology, control switch transistor to open and close time ratio, stable output voltage of a power supply. Early switch has a lower frequency, and the cost is higher, only applied in the field of satellite power of individual. Later research of the phase control switch power supply and the discrete component was used to construct various kinds of switching power supply, there are low switching frequency, complex circuit and control such complicated defects. With the continuous development of integrated circuit design and manufacturing technology, produced the different types of switching power supply chip, has greatly increased the frequency of switch power supply, enhance the performance of the switch. The current switching power supply adopts the advanced control theory and electronic components, efficient software, and greatly improve its own frequency stability, has been a rapid development..

\section{The development trend of low voltage switch electric appliances}

The operating mode diversification. a. The manual operation mode. The previous switch electrical appliances are mostly adopting manual operation mode. In structure design, in order to improve the contact points and closing speed, often use half has nothing to do with human operation. This way is completely depend on the handle directly applied to reach a certain threshold of a human operation. When the human more than threshold, unless the operator intentionally delay, otherwise will complete irrelevant on-off operation. Therefore, this operation mode in the actual operation can meet the requirements of contactor rapid opening and closing, is conducive to breaking overload current and short circuit current is connected. In the structural design of manual operation also has a variety of ways, mainly the following two aspects.

1) The installation position of manual operating mechanism. At present, the switch electrical appliances are designed according to requirements of the modular structure, therefore, through the combination of different installation, operating mechanism can separate module of its own in accordance with the requirements of different supporting assembly into different location of the operating mode, including the left (or right) positive operation, electrode positive operation and the left (or right) side operation, etc.

2) The manual handle position diversification. Manual operating handle generally has two rotational position, namely ON the position and OFF position, indicating the main circuit contactor 
in the ON or OFF state. In addition, the manual operating mechanism configuration handle can also has three operating position, namely, apart from the above two positions, adding a TEST position. When a controller will switch electrical appliances from broken position operation to test position, the auxiliary contact position change test, and the main circuit contact remain off state. Increase after the test position, the user can check in without power switch electrical appliances and other electrical equipment of electrical chain or control function.

b. The electric operating mode

Low voltage switch electric appliances while the majority with manual operation mode, but with the development of smart grid, to meet the requirements of remote control operation, including $\mathrm{ABB}$, famous foreign manufacturers began on the existing manual switch electrical derived electric operation. It is worth noting that the various modes of the installation of electric operating mechanism including installed behind the switch electrical appliances, positive, cupboard door, installed inside the cupboard door and embedded cupboard door installation, etc.

c. The automatic operating mode

If used in the production line of electric switch electrical operation with the corresponding industrial computer control, can automatically close break-brake operations according to technological requirements. Due to the switch with fuse appliances can achieve the purpose of overload and short circuit protection, so by this way can save a lot of investment, deeply user welcome. In addition, two electric operating switch electrical mount accessories mechanical chain connecting rod, deserve to go up again after the corresponding intelligent controller, can become a PC type automatic transfer switch, for normal or standby power automatic switching.

The structure design of safe and reliable. The structural design of low voltage switch electrical appliances are more safety and reliable. At present, the domestic and foreign manufacturers in product design measures mainly isolated position switch electric contact visible, contactor in closed position are not allowed to change the melt and external operating handle double insulation.

\section{The role of low voltage electrical switch}

Low voltage switch is used to connect or disconnect fewer than $1000 \mathrm{~V}$ AC and DC switch electrical appliances. Typically stretch arc or in air using long arc arcing grid divided into short arc. Commonly used low voltage switch knife switch, contactor, magnetic arc extinguishing device and automatic air switch. Contact resistance of switch electric contact not only with the contact itself of material, structure, mechanical force, such as voltage and current, is related to the working state of the switch electrical appliances. Switch electrical contact in the closed state, due to a current, is bound to cause fever, and fever has a certain influence on contact resistance. Contact in the state of on and off work, at the instant of the disconnect will appear arc, and arc of high temperature also affect the contact resistance.

\section{The low voltage electrical switch contact performance analysis}

In the process of the construction of low voltage electric switch, if the low voltage electrical contact pressure in the normal range, can pass to cross section area of the low voltage electric switch of determination methods for low voltage electric switch performance analysis. Specifically, in the process of analyzing the performance of low voltage electric switch, you can use a piece of carbon paper folding operation, and place the carbon folding operation in low voltage electric switch between dynamic contact and moving contact, through the use of low voltage electric switch closed, after a period of time, will be left current by mark on carbon paper. At this point, can appear on the carbon paper traces of low voltage electric switch contact performance analysis work, using reasonable calculation of the trace of area is calculated, can analysis to calculate the performance of low voltage electric switch contact. 


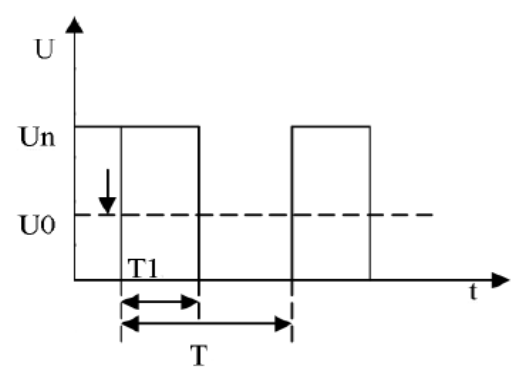

Fig. 1: Wide adjustable switch regulated power supply control principle

\section{The design of low voltage electrical switch}

The electric circuit and bus coupler circuit. As the basis of low-voltage distribution system, should be used with the function of communication framework of intelligent circuit breaker (regardless of the individual application system capacity is very small in the case of molded case circuit breaker), can easily realize the loop "four control", namely, telemetry, remote adjustment, remote control, remote communication. The current frame type intelligent circuit breaker has become mainstream, its built-in protection, alarm, intelligent controller set measurement, maintenance, operation and management, and other functions, various types of adjustable parameters online. Besides the protective function of electric power parameter based on frame type circuit breaker may also participate in the distribution management and control functions, such as the current unloading function, ZSI regional selective interlock function (such as CW2, CW3), additional automatic power transfer system, etc. Different from electric circuit can choose corresponding intelligent controller, and each type of intelligent controller has the basic function and optional features, can satisfy matching. Defect is on the market most intelligent frame type circuit breaker adopts common built-in current transformer protection and measurement, the precision is limited, caused power constrained parameter measuring precision, normally only at 1.0 level or less, in the occasion of measurement precision grade 0.5 and above applications, should the other configuration measuring current transformer and measure function of intelligent electric meter instead of circuit breaker, such as timely development frame type circuit breaker with high precision current transformer, an important performance is equivalent to the circuit breaker, and also saves considerable costs for end users. Intelligent circuit breaker and instrument of all the parameters can be read from a PC, achieve the following goals: overall operation fully controllable; the power quality and equipment data transparency; Power cuts, accident trip, the cause of the problem can be analyzed. As an important part of power distribution system, the electric circuit and bus coupler circuit can short message module, additional configuration for monitoring intelligent frame type circuit breaker operation state, when the circuit breaker preset incident, send a short message to one or more cell phone prompt the user timely, events of default can be all kinds of fault tripping abnormal alarm and power parameters.

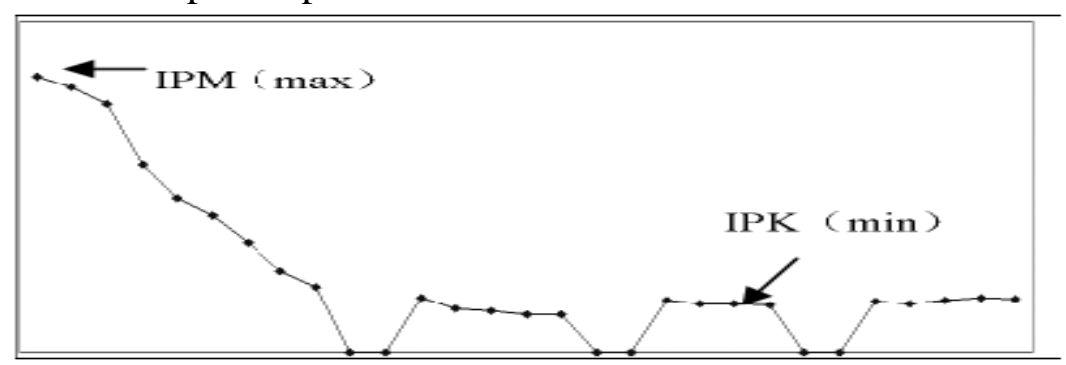

Fig. 2: The changes before and after the period of the noise voltage amplitude change trend

The feed loop. Feeder circuit should be reasonable allocation according to the control and monitoring requirements of different components, to meet the requirements of power distribution system overall (2 away, three remote, four remote). Capacity is larger, the circuit is relatively important, generally should realize the "four control", similar to the electric circuit, should configure intelligent frame type circuit breaker (such as CW1, CW2, CW3), and select suitable 
intelligent controller. On the occasion of measurement precision grade 0.5 and above applications, should the other configuration measuring current transformer and intelligent electric meter. Is more occasions circuit capacity is small, in accordance with the requirements of different monitoring configuration intelligent degree of different components can realize "four control" function in whole or in part.

(1) To choose smart molded case circuit breaker (such as CM1Z, CM2Z) and electric operating mechanism, display module, switch, when need to remote control, the switch is in the remote control, remote control switch, then through the upper machine remote parameter back road, upload the switch state and three phase current, three-phase voltage and other information, which can realize "four control", namely, telemetry, remote adjustment, remote control, remote communication; When need local control switch can be placed on the position, the function of remote control, remote sensing, remote adjustment and remote communication function, can be displayed through the module on the switch button to switch points closing operation.

(2) In measuring accuracy grade 0.5 and above, choose ordinary molded case circuit breaker (such as CM1 and CM2, CM3) and electric operating mechanism, configuration of measuring current transformer and intelligent electric meter. Intelligent electric meter by the field bus communication interface to access not only can measure the power parameters, can also configure the intelligent electric meter $\mathrm{I} / \mathrm{O}$ module is used to control the circuit breaker closing, acquisition switch state such as switch amount, so as to realize "three remote", namely, telemetry, remote control, remote communication.

\section{Summary}

To sum up, in the process of for the design of low voltage electric switch, combined with the actual situation, to the fully considering the using range of low voltage electric switch in the process of the performance test of low voltage electric switch and reflects the performance of the basis, scientifically designed for low voltage electric equipment switch, ensure that the use of low voltage electrical switch performance.

\section{References}

[1] Marc Bouissou, Jean-Louis Bon. A new formalism that combines advantages of fault-trees and Marko models: Boolean logic driven Markov processes. Reliability Engineering and System Safety. 2013

[2] X.J. Ma, X.B. Cao, G.L. Zhao. Design and analysis of the reliability of on-board computer system based on Markov-model. Joumal of Harbin Institute of Technology. 2012

[3] Takehisa Kohda, KoichiInoue. Probability Evaluation of System-Failure Occurrence Based on Minimal Cut-Sets. Reliability and Maintainability Symposium, 2002. Proceedings, Annual. 2012

[4] Fussell J.B. Fault tree analysis-concepts and technique. Generic Techniques in System Reliability Assesment. 2011. 